\title{
Acción de las radiaciones U.V. sobre el poli (cloruro de vinilo) flexible
}

\author{
JUAN J. ORTEGA, Dr. en Quimica Industrial \\ MANUEL BLANCO, Dr. en Ciencias Quimicas \\ ANGEL CUEVAS, Ledo. en Ciencias Quimicas \\ Laboratorio Central de Estructuras y Materiales. CDX-MOPU. Area de Materiales Orgánicos
}

\section{$R E S U M E N$}

En este trabajo se trata de poner de manifiesto el efecto de las radiaciones $U$. V. sobre el poli (cloruro de vinilo) plastificado, utilizado en forma de lámina para la impermeabilización, tanto en la Edificación como en la Obra Pública. La acción del envejecimiento por radiaciones $U . V$. se ha seguido a lo largo de 3.000 horas, comprobando las alteraciones producidas en la composición del material, caracteristicas mecánicas y estructura microscópica.

\section{$S U M M A R Y$}

The aim of this work is the study of the U.V. radiations effects on plasticized Poly (vinyl chloride) used as waterproofing membrane in Building and also in Public Works.

The ageing fact due to $U$.V. radiations has been followed for 3.000 hours, checking the changes produced in the composition of the material, on its mechanical characteristics and microscopic structure.

\section{N T R O D U C CIO N}

La tendencia actual de la investigación en el campo de la impermeabilización con materiales plásticos y elastoméricos se basa, fundamentalmente, más en la experimentación de los ya existentes, donde se encuentran comercializados más de un centenar que en la búsqueda de nuevos materiales $(1,2)$.

Las nuevas lineas de investigación tienden a comprobar la durabilidad del material con ensayos a largo plazo, terreno que está, prácticamente, inexplorado, asi como relacionar los resultados de dichos ensayos con los considerados de corto plazo (3).

Las pruebas a tener en cuenta son las de envejecimiento, en las que el material se somete a una serie de ensayos en condiciones de intemperie, laboratorio y obra.

Para evaluar su comportamiento se precisa fijar, de antemano, unas características, tales como: propiedades mecánicas, análisis químico y estudio microscópico.

En un principio, se pueden considerar dos tipos de envejecimiento:

- Natural.

- Artificial.

El envejecimiento artificial, que es una prueba de las consideradas como de "corto plazo", puede, a su vez, dividirse en los siguientes tipos:

- térmico; 
- arco de carbón;

- arco de xenón;

- radiaciones $\mathrm{U} . \mathrm{V}$.;

- ozono.

\section{PARTE EXPERIMENTAL}

De los ensayos clásicos de envejecimiento acelerado (arco de carbón, arco de xenón y radiaciones U.V.), las experiencias realizadas, tanto a nivel nacional como internacional, han puesto de manifiesto que para este tipo de materiales las pruebas deben efecturse mediante exposición a radiaciones U.V., por ser más rápido el deterioro del material.

Ya seleccionado el tipo de envejecimiento, se eligió como material para la investigación el poli (cloruro de vinilo) plastificado por ser, actualmente, el más empleado tanto para la impermeabilización en edificación (membranas de simple cobertura) como en la obra pública (geomembranas).

El proceso de envejecimiento es un fenómeno degradativo debido a reacciones homolíticas o radicálicas, al que habria que sumar, en el caso particular del poli (cloruro de vinilo), la degradacion por deshidrocloración de la macromolécula. La misión de la mayoría de los aditivos es parar o, al menos, reducir en lo posible dichos procesos degradativos.

La acción del envejecimiento por U.V. se ha seguido por los tres caminos siguientes:

\section{$\left.1^{\circ}\right)$ Alargamiento en rotura}

Al realizar el envejecimiento de las láminas de poli (cloruro de vinilo) plastificado durante $500 \mathrm{~h}$ a $50^{\circ} \mathrm{C}$, hemos tenido la ocasión de comprobar que los datos de degradación obtenidos, evaluados por sus características mecánicas, no permitían diferenciar láminas preparadas para la intemperie de aquellas que no llevaban tal preparación. Ello nos condujo a elevar el tiempo de exposición, al menos, a $3.000 \mathrm{~h}$ y aumentar, asimismo, la temperatura de trabajo a $70^{\circ} \mathrm{C}$, siempre con un programa de operación U.V./inmersión en agua.

T A B LA I

Variación del alargamiento en rotura en función del tiempo de envejecimiento a $70^{\circ} \mathrm{C}$

\begin{tabular}{|l|c|c|c|c|}
\hline \multicolumn{1}{|c|}{ TIPO DE LAMINA } & \multicolumn{3}{|c|}{ VARIACION DEL ALARGAMIENTO \% } \\
\cline { 2 - 4 } & $\mathbf{1 . 0 0 0} \mathbf{~}$ & $\mathbf{1 . 5 0 0} \mathbf{~}$ & $\mathbf{2 . 0 0 0} \mathbf{~}$ & $\mathbf{3 . 0 0 0} \mathbf{~ h}$ \\
\hline 1. Preparada para la intemperic & 1,5 & 1,5 & 7,1 & 8,5 \\
2. Resistente al betún & 15,0 & 42,6 & 43,9 & 44,0 \\
3. No preparada para la intemperie, pero revestida & 9,6 & 13,4 & $-18,5$ & 18.5 \\
4. Preparada para la intemperic & 1,0 & 5,0 & 6,5 & 8.7 \\
5. No preparada & 8,4 & 15,6 & 20,4 & 21.0 \\
6. No preparada para la intemperie & 14,0 & 22,3 & 23,3 & 27.7 \\
7. Preparada, con inserción de fibra de vidrio & -3.5 & 3,6 & 3.7 & 4.2 \\
\hline
\end{tabular}


Dicho programa de U.V./inmersión en agua se siguió como consecuencia de experiencias previas, donde se comprobó que exposiciones de $500 \mathrm{~h}$ a $50^{\circ} \mathrm{C}$ no afectaban apreciablemente a los materiales ensayados. Las láminas se someten a ciclos de 3 días de exposición a las radiaciones U.V. y otros tantos de inmersión en agua.

En las condiciones de $70^{\circ} \mathrm{C}$, los resultados ya eran más claros; se podia observar qué láminas gozaban de una preparación adecuada para la intemperie (las señaladas con los números $1,4 \mathrm{y}$ 7 de la tabla I) y cuáles precisaban de una protección (el resto de las ensayadas). En la mencionada tabla se presentan los datos obtenidos en cuanto a la variación del alargamiento en rotura, en función del tiempo de exposición de la lámina. Cabe destacar que al cabo de las $1.500 \mathrm{~h}$ de exposición el efecto degradativo está perfectamente localizado.

\section{$\left.2^{\circ}\right)$ Composición}

Además de las propiedades mecánicas, se ha seguido el efecto del envejecimiento de las láminas a lo largo del ensayo en función de la composición de las mismas, siguiendo el procedimiento que se cita a continuación:

\section{Separación de componentes}

Se pesa $1 \mathrm{~g}$ de lámina y se pasa a un extractor Soxhlet; en el matraz correspondiente se han colocado $120 \mathrm{ml}$ de éter etílico; al cabo de las $6 \mathrm{~h}$, los plastificantes se habrán extraído; de la parte etérea se calcula el contenido en plastificantes.

Al resto del material, que queda en el cartucho del Soxhlet, se le agragan $20 \mathrm{ml}$ de 1,1,2,2,-tetracloroetano y se calienta, con el fin de disolver la resina; se añaden $20 \mathrm{ml}$ de tetrahidrofurano y se pasan a una centrifuga durante 30 minutos. A continuacion se decanta la solución, y al resto sólido, se le añaden otros $20 \mathrm{~m} l$ de tetrahidrofurano y se repite el centrifugado; de nuevo se decanta, y la parte sólida se calienta a $110^{\circ} \mathrm{C}$ una hora, se enfría, se pesa y se calculan los estabilizadores y fillers. El contenido en resina se calcula por diferencia.

\section{Identificación de los componentes}

La identificación de los componentes del poli (cloruro de vinilo) se ha llevado a cabo por espectroscopia IR.

El espectro de la figura 1 muestra el plastificante de la lámina 6, donde se pueden observar, como bandas características, la de $1.712 \mathrm{~cm}^{-1}$ de tensión $\mathrm{C}=0$ desplaza por la conjugación del anilllo aromático; $1.263 \mathrm{~cm}^{-1}$ tensión asimétrica de la agrupación C-O-C y a $1.119 \mathrm{~cm}^{-1}$ la tensión simétrica de la misma agrupación, por lo que concuerda con el ftalato de dioctilo.

En la figura 2 se muestra el espectro correspondiente a los fillers y estabilizadores (cargas inorgánicas) de la lámina. Como el espectro se ha llevado a cabo utilizando nujol como disolvente, éstas son las bandas más llamativas que aparecen en el mismo, siendo de destacar la que se encuentra a $869 \mathrm{~cm}^{-1}$, atribuible a una carga de carbonato cálcico.

En la figura 3 se presenta el espectro correspondiente a las resinas de poli (cloruro de vinilo). La pequeña banda situada a $1.720 \mathrm{~cm}^{-1}$, propia del grupo carbonilo, podría deberse a que la resina de PVC va asociada a poli (acetato de vinilo) o a una mala separación del plastificante. Nos inclinamos por el primero de los hechos, ya que la banda que aparece a $1.030 \mathrm{~cm}$ । confirma la existencia de la agrupación acetato. 


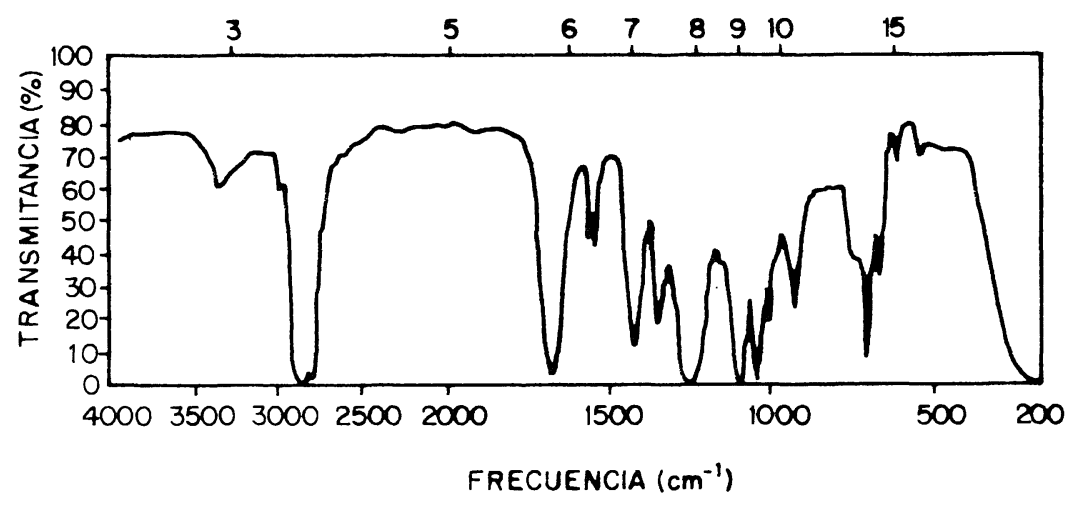

Fig. 1.-Espectro infrarrojo de los plastificantes de la lámina 6

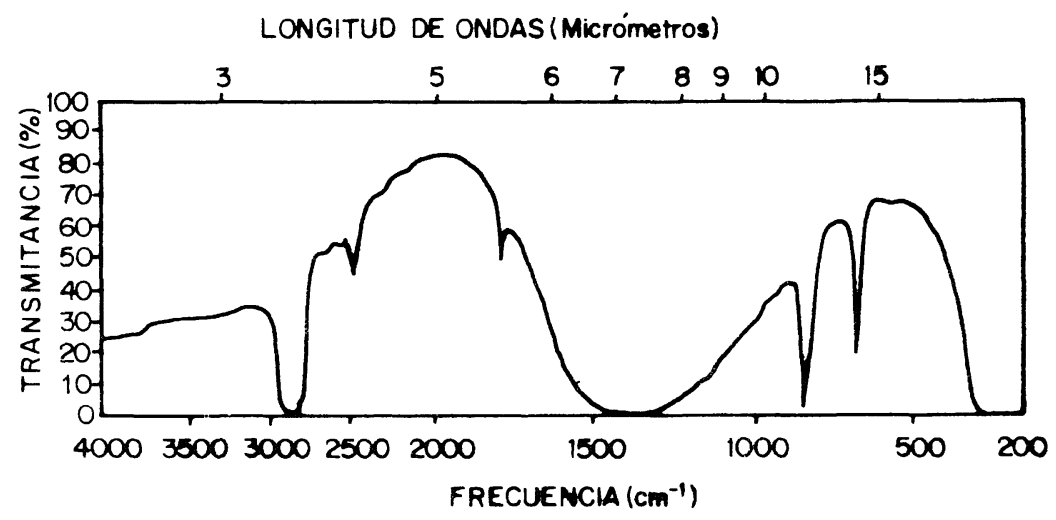

Fig. 2.-Espectro IR del filler y cargas inorgánicas.

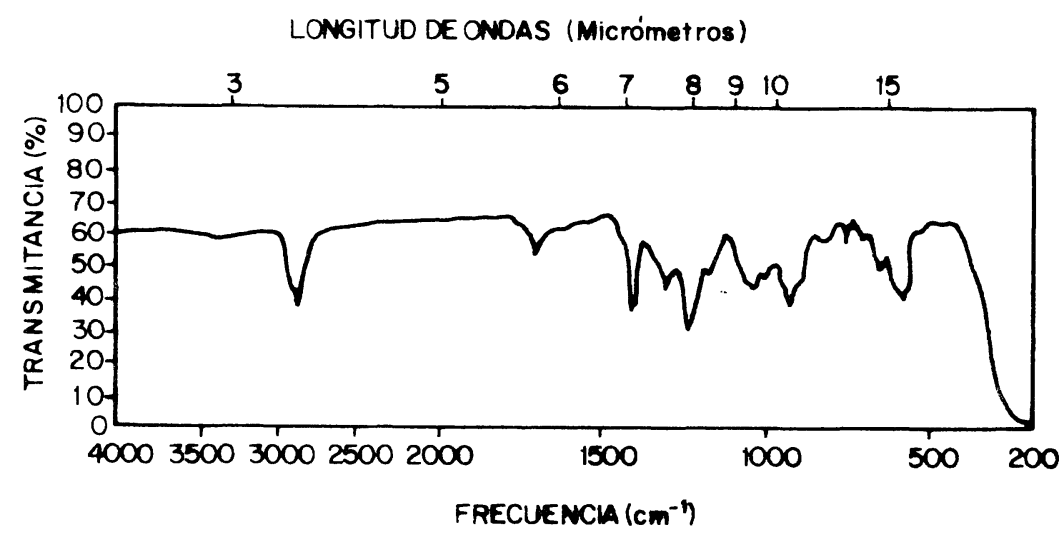

Fig. 3.-Espectro IR de la resina.

En la tabla II se presentan los datos de composición de las láminas número 1 (preparada para la intemperie) y n.o 6 (no preparada para la intemperie), en cuanto a lo que se refiere a plastificantes, filler y resinas. 
TABLA II

Composición de lámina de PVC plastificado antes y después de su envejecimiento

COMPOSICION \%

\begin{tabular}{|c|c|c|c|c|c|c|c|}
\hline \multirow{2}{*}{$\begin{array}{l}\text { Tipo de } \\
\text { lámina }\end{array}$} & \multicolumn{3}{|c|}{ Original } & \multicolumn{3}{|c|}{ Envejecida 3.000 h } & \multirow{2}{*}{$\begin{array}{c}\text { Pérdida } \\
\text { de plás- } \\
\text { tificante, } \\
\%\end{array}$} \\
\hline & Plástificante & Filler & Resina & Plastificante & Filler & Resina & \\
\hline Preparada intemperie & 35,76 & 13,78 & 50,46 & 33,79 & 14,06 & 52,15 & 6 \\
\hline No prep. intemperie & 26.28 & 19,32 & 54,40 & 19,34 & 21,49 & 59,17 & 26 \\
\hline
\end{tabular}

Los datos se refieren a láminas originales y después de un envejecimiento en las condiciones antes citadas durante $3.000 \mathrm{~h}$. Se puede observar que en la lámina n. ${ }^{\circ}$, la pérdida de plastificante es del $6 \%$, mientras que en la n.o 6 alcanza hasta un valor del $26 \%$. Esto nos confirma los resultados que obteniamos de pérdidas de alargamiento, ya que la n. 1 presentaba, al cabo de las $3.000 \mathrm{~h}$, una disminución del $8,5 \%$, mientras que la n. 6 lo hacia en un $27,7 \%$.

\section{3. ${ }^{\circ}$ Análisis microscópico}

Por último, la comprobación del deterioro del poli (cloruro de vinilo) se ha seguido con ayuda de la microscopia clectrónica de barrido, que vino a confirmar los datos obtenidos de propiedades mecánicas y composición. Las fotografias de la figura 4 , muestran la lámina $n .^{\circ} 1$, por su cara gris, en su estado original y envejecidas a las $1.500,2.000$ y 3.000 horas.
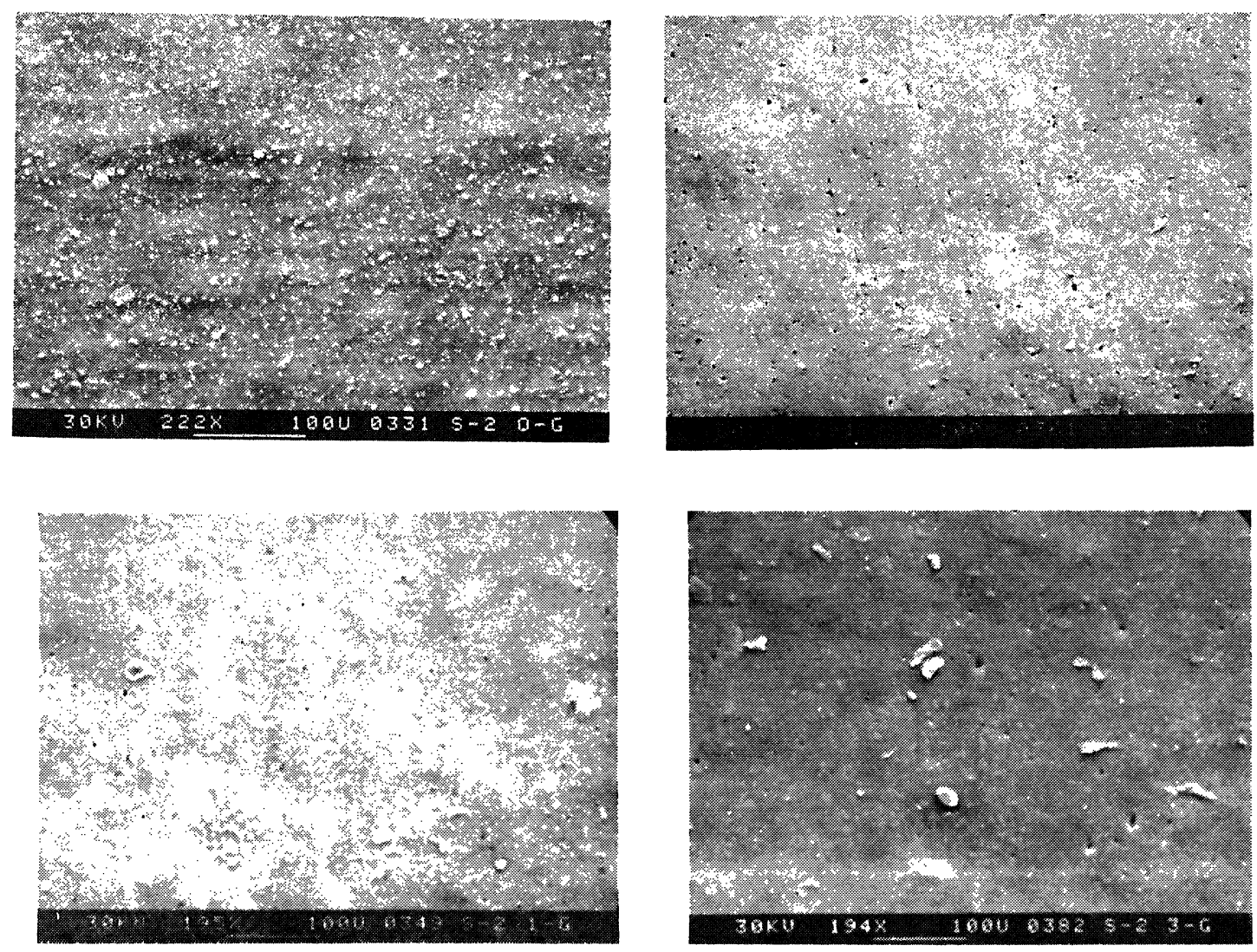

IFig. 4 
En la figura 5 se presentan las fotografias correspondientes a la estructura de la lámina n. ${ }^{\circ} 1$ por su cara blanca expuesta directamente a la luz U.V. Aquí, la acción de las radiaciones es mucho más acusada, debido, por un lado, a la exposición directa a la mencionada luz $\mathrm{y}$, por otro, a la ausencia de negro de humo, que diminuiría el proceso degradativo.
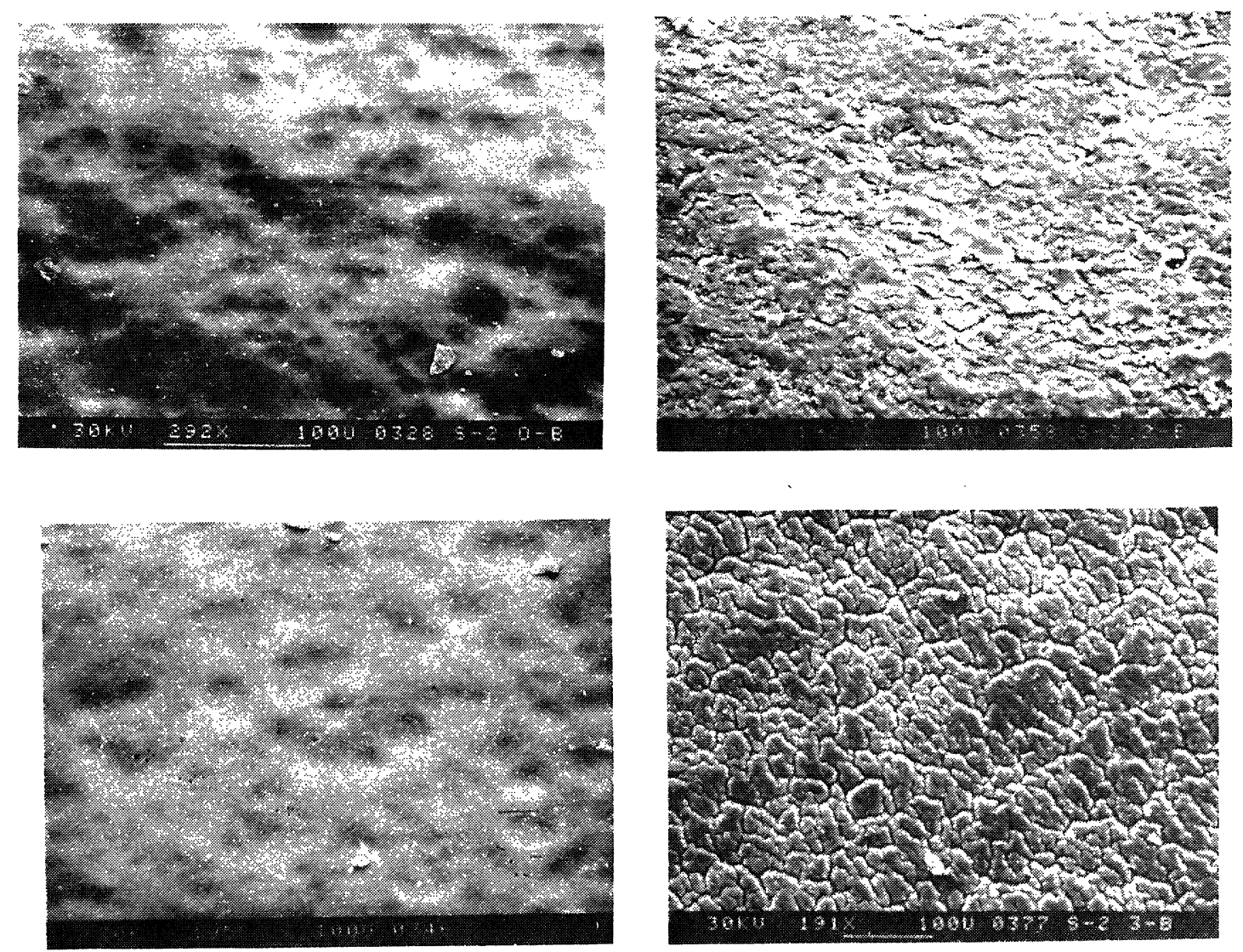

Fig. 5

\section{CONCLUSIONES}

- Láminas de poli (cloruro de vinilo) plastificado preparadas y no preparadas para su aplicación directa a la intemperie pueden diferenciarse a partir de las $1.500 \mathrm{~h}$ de envejecimiento, como se prueba con los resultados de pérdida de alargamiento. Todas las láminas preparadas no llegan a perder el $10 \%$ de esta característica.

- La disminución del alargamiento en rotura va acompañada de una pérdida de plastificante, siendo ésta mucho más importante cuando las láminas no llevan los aditivos necesarios para su utilización en exposición directa a las radiaciones solares.

- La microscopia electrónica pone de manifiesto, de una manera clara, la degradación del material en función del tiempo de exposición a las radiaciones U.V.

\section{B I B L I O G R A F I A}

(1) ROSSITER, W. J. Jr. y MATHEY, R. G.: "Elastomeric Roofing: A Survey". National Bureau of Standards, Department o Commerce, Washington. 1979.

(2) GAMSKI, K.: "Presentation des membranes souples d'etancheite”. Memoires C.E.R.E.S., n." 55. Lieja, 1980.

(3) MARTIN VICENTE, L.: SANTOLINO MARTIN. S. y JORGE TAPIA, G.: Rev. Plast. Mod. 40. n." 292 (1980). 465-471. 